Pacific Journal of Mathematic 


\title{
COMMUTATIVITY IN LOCALLY COMPACT RINGS
}

\author{
JAMES B. LUCKE
}

\begin{abstract}
A structure theorem is given for all locally compact rings such that $x$ belongs to the closure of $\left\{x^{n}: n \geqq 2\right\}$, in particular, all such rings are commutative, a result which extends a wellknown theorem of Jacobson. Similarly we show the commutativity of semisimple locally compact rings satisfying topological analogues of properties studied by Herstein.
\end{abstract}

Jacobson has shown that a ring is commutative if for every $x$ there is some $n(x) \geqq 2$ such that $x^{n(x)}=x$ [5, Th. 1, p. 212]. Herstein has generalized this result, and certain of his and other generalizations are of interest here. A ring is commutative if (and only if) for all $x$ and $y$ there is some $n(x, y) \geqq 2$ such that $\left(x^{n(x, y)}-x\right) y=y\left(x^{n(x, y)}-x\right)$ [4, Th. 2]; a ring is commutative if (and only if) for all $x$ and $y$ there is some $n(x, y) \geqq 2$ such that $x y-y x=(x y-y x)^{n(x, y)}$ [3, Th. 6]; a semisimple ring is commutative if (and only if) for all $x$ and $y$ there is some $n(x, y) \geqq 1$ such that $x^{n(x, y)} y=y x^{n(x, y)}$ [4, Th. 1] or if for all $x$ and $y$ there are $n, m \geqq 1$ such that $x^{n} y^{m}=y^{m} x^{n}$ [1, Lemma 1]. The investigation of analogous conditions for topological rings is the major concern of this paper.

1. A topological analogue of Jacobson's condition. If $x^{n}=x$ for some $n \geqq 2$, then an inductive argument shows that $x^{k(n-1)+1}=x$ for all $k \geqq 1$. A possible topological analogue of Jacobson's condition would thus be that for every $x$ there is some $n(x) \geqq 2$ such that $\lim _{k} x^{k(n(x)-1)+1}=x$. But this implies that $x^{n(x)}=x$, since

$$
x^{n(x)}=x^{n(x)-1} x=x^{n(x)-1} \lim _{k} x^{k(n(x)-1)+1}=\lim _{k} x^{(k+1)(n(x)-1)+1}=x .
$$

Thus all topological rings having this property have Jacobson's property and hence are commutative.

A less trivial analogue of Jacobson's condition is that for every $x$ in the topological ring $A, x$ belongs to the closure of $\left\{x^{n}: n \geqq 2\right\}$. In our investigation of these rings, rings with no nonzero topological nilpotents play an important role. Recall that an element $x$ of a topological ring is a topological nilpotent if $\lim _{n} x^{n}=0$. We shall prove that a locally compact ring has no nonzero topological nilpotents if and only if it is the topological direct sum of a discrete ring having no nonzero nilpotents and a ring $B$ that is the local direct sum of a family of discrete rings having no nonzero nilpotents with respect to finite subfields. From this it is easy to derive a structure theorem for locally compact rings 
having the topological analogue of Jacobson's property mentioned above.

LEMMA 1. If $A$ is a locally compact ring with no nonzero topological nilpotents, then $A$ is totally disconnected.

Proof. The connected component $C$ of zero in $A$ is a closed ideal of $A$ and so is itself a connected locally compact ring with no nonzero topological nilpotents. By hypothesis, $C$ is not annihilated by any of its nonzero elements, for if $x C=(0)$, then $x^{2}=0$, so $x=0$. Thus $C$ is a finite-dimensional algebra over the real numbers (cf. [6, Th. III]). As the radical of a finite-dimensional algebra is nilpotent, $C$ is a semisimple algebra. If $C \neq(0)$, then by Wedderburn's Theorem, $C$ has an identity $e$, and clearly $(1 / 2) e$ would then be a nonzero topological nilpotent contrary to our hypothesis. Thus $C=(0)$, and so $A$ is totally disconnected.

Lemma 2. A compact ring $A$ has no nonzero topological nilpotents if and only if $A$ is the Cartesian product of finite fields.

Proof. Necessity: By Lemma 1, $A$ is totally disconnected. Thus the radical $J(A)$ of $A$ is topologically nilpotent [11, Th. 14], and hence is the zero ideal. Thus $A$ is a compact semisimple ring, and so $A$ is topologically isomorphic to the Cartesian product of a family of finite simple rings [11, Th. 16]. A finite simple ring is a matrix ring over a finite field, and unless the matrix ring is just the finite field itself, it will have nonzero nilpotent elements. Thus as $A$ has no nonzero nilpotents, $A$ is topologically isomorphic to the Cartesian product of a family of finite fields. Sufficiency: Clearly zero is the only topological nilpotent in the Cartesian product of a family of finite fields.

Lemma 3. If $A$ is a ring with no nonzero nilpotents, then every idempotent is in the center of $A$.

Proof. If $e$ is an idempotent and if $a \in A$, an easy calculation shows that $(a e-e a e)^{2}=0$, hence $a e-e a e=0$. Similarly, $e a=e a e$ and thus $a e=e a$.

We recall that the local direct sum of a family $\left(A_{\gamma}\right)_{\gamma \in \Gamma}$ of topological rings with respect to open subrings $\left(B_{\gamma}\right)_{r \in \Gamma}$ is the subring of the Cartesian product $\Pi_{\gamma} A_{r}$ consisting of all $\left(a_{\gamma}\right)$ such that $a_{\gamma} \in B_{\gamma}$ for all but finitely many $\gamma$, topologized by declaring all neighborhoods of zero in the topological ring $\Pi_{r} B_{\gamma}$ to be a fundamental system of neighborhoods of zero in the local direct sum. It is easy to see that the local direct sum equipped with this topology is indeed a topological ring. 
THEOREM 1. A locally compact ring A has no nonzero topological nilpotents if and only if $A$ is the topological direct sum of a discrete ring having no nonzero nilpotents and a ring $B$ (possibly the zero ring) that is topologically isomorphic to the local direct sum of a family of discrete rings having no nonzero nilpotents with respect to finite subfields.

Proof. Necessity: As $A$ is totally disconnected by Lemma $1, A$ contains a compact open subring $F$ [7, Lemma 4]. By Lemma 2, $F$ is topologically isomorphic to the product of finite fields. Consequently there exists in $F$ a summable orthogonal family $\left(e_{\gamma}\right)_{\gamma \in \Gamma}$ of idempotents such that $F e_{\gamma}$ is a finite field and $\sum_{\gamma \in \Gamma} e_{\gamma}=e$, the identity of $F$.

By Lemma 3, $e$ is in the center of $A$, so $A e$ and $A(1-e)=\{a-a e$ : $a \in A\}$ are ideals. The continuous mappings $a \rightarrow a e$ and $a \rightarrow(a-a e)$ are the projections from $A$ onto $A e$ and $A(1-e)$. Thus $A$ is the topological direct sum of $A e$ and $A(1-e)$. As $e$ is the identity of $F, F \cap A(1-e)=(0)$. Thus as $F$ is open, $A(1-e)$ is discrete and hence has no nonzero nilpotents.

As $F$ is open and as $A e_{\gamma} \cap F=F e_{\gamma}$, a finite field, $A e_{\gamma}$ is discrete and is an ideal as $e_{\gamma}$ is in the center of $A$. Consequently $A e_{\gamma}$ has no nonzero nilpotents. It will therefore suffice to show that $B=A e$ is topologically isomorphic to the local direct sum of the descrete rings $A e_{r}$, with respect to the finite subfields $F e_{\gamma}$.

Let $B^{\prime}$ be the local direct sum of the $A e_{\gamma}$ 's with respect to the $F e_{\gamma}$ 's. Let $K: b \rightarrow\left(b e_{\gamma}\right) \in \Pi_{\gamma} A e_{\gamma}$. Clearly $b \rightarrow b e_{\gamma}$ is a continuous homomorphism for each $\gamma$, hence $K$ is a continuous homomorphism from $B$ into $\prod_{\gamma} A e_{\gamma}$. If $b \in B$, then $\left(b e_{\gamma}\right)$ is summable and $\sum_{\gamma} b e_{\gamma}=b\left(\sum_{\gamma} e_{\gamma}\right)=b e=b$. Therefore as $F$ is open in $B, b e_{\gamma} \in F \cap A e_{\gamma}=F e_{\gamma}$ for all but finitely many $\gamma \in \Gamma$. Thus $K(B) \cong B^{\prime}$.

The mapping $K$ is an isomorphism onto $K(B)$, since if $x \in B$ and if $x e_{\gamma}=0$ for all $\gamma \in \Gamma$, then $x=x e=x\left(\sum_{\gamma} e_{\gamma}\right)=\sum_{\gamma} x e_{\gamma}=0$. Let $y_{\beta} \in F e_{\beta}$, and let $x_{\gamma}=0$ for all $\gamma \neq \beta, x_{\beta}=y_{\beta}$; then $\left(x_{\gamma}\right)=K\left(y_{\beta}\right) \in K(F)$ since $\left(e_{\gamma}\right) \gamma$ is an orthogonal family. Thus $K(F)$ contains a dense subring of $\Pi_{\gamma} F e_{\gamma}$, and hence $K(F)=\Pi_{\gamma} F e_{\gamma}$ as $K(F)$ is compact. As the restriction of $K$ to $F$ is thus a continuous isomorphism from conpact $F$ onto $\prod_{\gamma} F e_{\gamma}$, $F$ is topologically isomorphic to $\Pi_{\gamma} F e_{\gamma}$ under $K$.

Thus it sufficices to show that $K(B) \supseteqq B^{\prime}$, for $K$ is then, by the definition of the local direct sum, a topological isomorphism from $B$ onto $B^{\prime}$. If $\left(b_{\gamma} e_{\gamma}\right) \in B^{\prime}$, then $b_{\gamma} e_{\gamma} \in F e_{\gamma}$ for all but finitely many $\gamma$, say $\gamma_{1}, \cdots, \gamma_{n}$. Call this set $\Gamma_{1}$ and let $\Gamma-\Gamma_{1}=\Gamma_{2}$. Thus $\sum_{\gamma \in \Gamma_{1}} b_{\gamma} e_{\gamma} \in B$ and $b_{\gamma} e_{\gamma} \in F$ for all $\gamma \in \Gamma_{2}$. Hence as $F$ is topologically isomorphic to $\prod_{\gamma} F e_{\gamma}, b^{\prime}=\sum_{\gamma \in \Gamma_{2}} b_{\gamma} e_{\gamma} \in B$. Thus $b=b^{\prime}+\sum_{\gamma \in \Gamma_{1}} b_{\gamma} e_{\gamma} \in B$, and $b e_{\gamma}=b_{\gamma} e_{\gamma}$, so $K(b)=\left(b_{\gamma} e_{\gamma}\right)$. The sufficiency is clear. 
We will call a ring $A$ a Jacobson ring if given any $x \in A$ there is an $n(x) \geqq 2$ such that $x^{n(x)}=x$. All Jacobson rings are commutative [5, Th. 1, p. 212], and in extending this result to topological rings we give the following definition, noting that it reduces to Jacobson's condition in the discrete case.

Definition. A topological ring $A$ is a $J$-ring if for each $x \in A, x$ belongs to the closure of $\left\{x^{n}: n \geqq 2\right\}$.

Lemma 4. If $A$ is a J-ring, then $A$ has no nonzero topological nilpotents.

Proof. If $\lim _{n} x^{n}=0$, then since $x$ belongs to the closure of $\left\{x^{n}: n \geqq 2\right\}$, we conclude that $x=0$.

Theorem 2. A locally compact ring $A$ is a J-ring if and only if $A$ is the topological direct sum of a discrete Jacobson ring and a ring $B$ which is topologically isomorphic to the local direct sum of a family of discrete Jacobson rings with respect to finite subfields.

Proof. Necessity: By Theorem 1 and Lemma 4, $A$ is the topological direct sum of a discrete ring $C$ and a ring $B$ which is topologically isomorphic to the local direct sum of a family of discrete rings with respect to finite subfields. As each of these rings is an ideal of $A$, each is a discrete $J$-ring and so is a Jacobson ring.

Sufficiency: Let $B$ be the local direct sum of a family of discrete Jacobson rings $B_{r}, \gamma \in \Gamma$ with respect to finite subfields $F_{r}, \gamma \in \Gamma$. Let $\left(x_{\gamma}\right) \in B$ and let $U$ be a neighborhood of zero in $B$. Then we may assume that there is a finite subset $\Delta$ of $\Gamma$ such that $x_{\gamma} \in F_{\gamma}$ for all $\gamma \notin \Delta$ and $U=\prod_{\gamma} G_{\gamma}$, where $G_{\gamma}=F_{\gamma}$ for all $\gamma \notin \Delta$. For each $\gamma \in \Delta$, let $n(\gamma)>1$ be such that $x_{\gamma}^{n(\gamma)}=x_{\gamma}$. Let $n=1+\Pi_{\gamma \in \Delta}(n(\gamma)-1)$. An inductive argument shows that $x_{\gamma}^{n}=x_{\gamma}$ for all $\gamma \in \Delta$. Hence $\left(x_{\gamma}\right)^{n}-$ $\left(x_{\gamma}\right) \in U$. Thus $B$ is a $J$-ring, and consequently $A$ is also a $J$-ring.

As all Jacobson rings are commutative we have the following analogue of Jacobson's Theorem:

Corollary. A locally compact J-ring is commutative.

THEOREM 3. A locally compact ring $A$ is a Jacobson ring if and only if there exists $N \geqq 2$ such that $A$ is the topological direct sum of a discrete Jacobson ring and a ring $B$ that is topologically isomorphic to the local direct sum of a family of discrete Jacobson rings with respect to finite subfields of order $\leqq N$. 
Proof. Necessity: Let $\left|B_{\gamma}\right|=$ the order of $B_{\gamma}$. By Theorem 2 it suffices to show that $\sup \left|B_{r}\right|<+\infty$. If $\sup \left|B_{r}\right|=+\infty$, then there exists $\left(x_{\gamma}\right) \in \prod_{r} B_{r}$ such that the orders of the $x_{r}$ 's are unbounded. Consequently for no $n$ does $x_{\gamma}^{n}=x_{\gamma}$ for all $\gamma$, i.e., for no $n$ does $\left(x_{\gamma}\right)^{n}=\left(x_{\gamma}\right)$.

Sufficiency: Let $\left(A_{\gamma}\right)_{\gamma \in \Gamma}$ be a family of discrete Jacobson rings with finite subfields $B_{\gamma}$ such that $\left|B_{\gamma}\right| \leqq N$ for all $\gamma$. Let $\left(x_{\gamma}\right)$ be in the local direct sum of the $A_{r}$ 's with respect to the $B_{r}$ 's. There exists a finite subset $\Delta$ of $\Gamma$ such that if $\gamma \notin \Delta, x_{\gamma} \in B_{\gamma}$. Since each $A_{r}$ is a Jacobson ring, for $\gamma \in \Delta$ there is $n(\gamma)$ such that $x_{\gamma}^{n(\gamma)}=x_{\gamma}$.

If $x_{\gamma}^{n(\gamma)}=x_{\gamma}$, an inductive argument shows that $x_{\gamma}^{k(n(\gamma)-1)+1}=x_{\gamma}$ for all $k$. If $x_{\gamma} \in B_{\gamma}$, then $\left|B_{\gamma}\right| \leqq N$, so since $\left|B_{\gamma}\right|-1<N$, $x_{\gamma}^{1+k(N !)}=x_{\gamma}$ for all $k$. Let $n=1+\left[(N !) \prod_{\gamma \in \Delta}(n(\gamma)-1)\right]$. Then $x_{\gamma}^{n}=x_{\gamma}$ for all $\gamma$, i.e., $\left(x_{\gamma}\right)^{n}=\left(x_{\gamma}\right)$.

2. Analogues of four of Herstein's results. An analogue for topological rings of the first of Herstein's conditions that are mentioned above is that for all $x$ and $y, x y-y x$ is in the closure of $\left\{x^{n} y-y x^{n}: \geqq 2\right\}$, and we say such a topological ring is an $H_{1}-r i n g$. An analogue of the second of Herstein's conditions is that for all $x$ and $y, x y-y x$ is in the closure of $\left\{(x y-y x)^{n}: n \geqq 2\right\}$, and we say such a topological ring is an $H_{2}$-ring. (If $(x y-y x)^{n(x, y)}=x y-y x$, then

$$
(x y-y x)^{k[n(x, y)-1]+1}=x y-y x
$$

for all $k \geqq 1$; hence another topological analogue is the assumption that for each $x, y \in A$, there exists $n(x, y) \geqq 2$ that $\lim _{k}(x y-y x)^{k[n(x, y)-1]+1}=$ $x y-y x$; however by an argument similar to that of the first paragraph of $\S 1$, this condition implies that $(x y-y x)^{n(x, y)}=x y-y x$.) Similarly an analogue of the third of Herstein's conditions is that for all $x, y$ in $A, \lim _{n} x^{n} y-y x^{n}=0$, and we say such topological rings are $H_{3}$-rings, just as we will call $H_{4}$-rings those topological rings in which for all $x, y$ there is an $m(x, y) \geqq 1$ such that $\lim _{n} x^{n} y^{m(x, y)}-y^{m(x, y)} x^{n}=0$. We shall prove that those $H_{i}-$ rings which are semisimple and locally compact are commutative, $i=1,2,3,4$.

Lemma 5. All idempotents in an $H_{i-r i n g, ~} i=1,2,3,4$, commute.

Proof. Let $e$ and $f$ be idempotents in such a ring $A$. Then $(e f e-e f)^{2}=0$, so $\left\{(e f e-e f)^{n} e-e(e f e-e f)^{n}: n \geqq 2\right\}=\{0\}$. Therefore, if $A$ is an $H_{1}$-ring, then $(e f e-e f) e-e(e f e-e f)=0$, so

$$
0=(e f e-e f) e=e(e f e-e f)=e f e-e f .
$$

If $A$ is an $H_{2}$-ring, then (ef)e-e(ef) $=e f e-e f=0$ since $e f e-e f$ is in the closure of $\left\{[(e f) e-e(e f)]^{n}: n \geqq 2\right\}=\{0\}$. Similarly in either case 
$e f e=f e$, so $e f=f e$. As $0=\lim _{n} e^{n} f-f e^{n}=\lim _{n} e^{n} f^{m}-f^{m} e^{n}=e f-f e$, the assention also holds for $H_{3}$ and $H_{4}$-rings.

Since it is clear that all subrings and quotient rings determined by closed ideals of $H_{i}$-rings are $H_{i}$-rings, $i=1,2,3,4$, and since all idempotents in such rings commute, we see that the following is applicable.

LEMMA 6. Let $P$ be a property of Hausdorff topological rings such that:

(1) if $A$ is a Hausdorff topological ring with property $P$, then every subring of $A$ has property $P$ and $A / B$ has property $P$ where $B$ is any closed ideal of $A$,

(2) if $A$ has property $P$, then all idempotents in $A$ commute. If $A$ is a locally compact primitive ring with property $P$, then $A$ is a division ring.

Proof. Since $A$ is a semisimple ring, $A$ is the topological direct sum of a connected ring $B$ and a totally disconnected ring $C$, where $B$ is a semisimple algebra over $R$ of finite dimension [7, Th. 2]. As $A$ is primitive, either $A=B$ or $A=C$. In the former case $A$ is a matrix ring since it is primitive, and so has idempotents which do not commute unless it is a division ring.

It suffices, therefore, to consider the case in which $A$ is totally disconnected. We shall first prove the assertion under the additional assumption that $A$ is a $Q$-ring (i.e., the set of quasi-invertible elements is a neighborhood of zero). We may consider $A$ to be a dense ring of linear operators on a vector space $E$ over a division ring $D$. If $E$ is not one-dimensional, then $E$ has a two-dimensional subspace $M$ with basis $\left\{z_{1}, z_{2}\right\}$. Let $B=\{a \in A: a(M) \subseteq M\}$, and let

$$
N=\{a \in A: a(M)=(0)\}=K_{1} \cap K_{2}
$$

where $K_{i}=\left\{a \in A: a\left(z_{i}\right)=0\right\}, i=1,2$.

There exists $u \in A$ such that $u\left(z_{1}\right)=z_{1}$, and hence $x-x u \in K_{1}$, for all $x \in A$. If $v \notin K_{1}$, then there exists $w \in A$ such that $w v\left(z_{1}\right)=z_{1}$, so as $u=w v+(u-w v)$ and $u-w v \in K_{1}, A=A u+K_{1}=A v+K_{1}$. Therefore $K_{1}$, and similarly $K_{2}$, is a regular maximal left ideal, an observation of the referee that simplifies the proof. Hence $K_{1}$ and $K_{2}$ are closed (cf. [11, Th. 2]), so $N$ is a closed ideal of $B$. By hypothesis $B / N$ is therefore a Hausdorff topological ring having property $P$. Thus all idempotents in $B / N$ commute; but $B / N$ is isomorphic to the ring of all linear operators on $M$, a ring containing idempotents which do not commute. Hence $E$ is one-dimensional and $A$ is a division ring.

Next we shall show that $A$ is necessarily a $Q$-ring, from which 
the result follows by preceding. As $A$ is totally disconnected $A$ has a compact open subring $D$ [7, Lemma 4]. If $D=J(D)$, the radical of $D$, then $D$ and hence $A$ are $Q$-rings. Assume therefore that $J(D) \subset D$. We shall show that $D / J(D)$ is a finite ring and hence is discrete.

The radical, $J(D)$, of $D$ is closed [8, Th. 1], $D / J(D)$ is compact semisimple ring and thus $D / J(D)$ is topologically isomorphic to the Cartesian product of a family $\left(F_{\gamma}\right)_{\gamma \in \Gamma}$ of finite simple rings with identities $\left(f_{\gamma}\right)_{\gamma \in \Gamma}[11$, Th. 16]. As $J(D)$ is topologically nilpotent [11, Th. 14], $D$ is suitable for building idempotents [12, Lemma 4] (cf. [11, Lemma 12]). Suppose that $\Gamma$ has more than one element, say $\{\alpha, \beta\} \subseteq \Gamma$. Then there are nonzero orthogonal idempotents $e_{\alpha}, e_{\beta}$ in $D$ such that $e_{\alpha}+J(D)$, $e_{\beta}+J(D)$ correspond, respectively, under the isomorphism to $\left(f_{\gamma}^{\alpha}\right),\left(f_{\gamma}^{\beta}\right)$ where $f_{\gamma}^{\lambda}=0 \in F_{\gamma}$ if $\gamma \neq \lambda$ and $f_{\lambda}^{\lambda}=f_{\lambda}$. Let $\phi$ be the canonical mapping $x \rightarrow x+J(D)$ from $D$ onto $D / J(D)$. As $\left(f_{\gamma}^{\alpha}\right)+\left(f_{\gamma}^{\beta}\right)$ annihilates the open neighborhood $\Pi_{\gamma \in \Gamma} G_{\gamma}$ of zero where $G_{\alpha}=\{0\}, G_{\beta}=\{0\}$, and $G_{\gamma}=F_{\gamma}$ for $\gamma \neq \alpha, \beta$, we conclude that $\phi\left(e_{\alpha}+e_{\beta}\right)$ annihilates a neighborhood $V$ of zero in $D / J(D)$. Consequently $U=\phi^{-1}(V)$ is a neighborhood of zero in $D$, and $\left(e_{\alpha}+e_{\beta}\right) U\left(e_{\alpha}+e_{\beta}\right) \subseteq J(D)$ (cf. [7, proof of Th. 11]). Therefore as $\left(e_{\alpha}+e_{\beta}\right) U\left(e_{\alpha}+e_{\beta}\right)=U \cap\left(e_{\alpha}+e_{\beta}\right) A\left(e_{\alpha}+e_{\beta}\right),\left(e_{\alpha}+e_{\beta}\right) U\left(e_{\alpha}+e_{\beta}\right)$ is a neighborhood of zero in $\left(e_{\alpha}+e_{\beta}\right) A\left(e_{\alpha}+e_{\beta}\right)$ consisting of quasi-invertable elements, so $\left(e_{\alpha}+e_{\beta}\right) A\left(e_{\alpha}+e_{\beta}\right)$ is a $Q$-ring. As $\left(e_{\alpha}+e_{\beta}\right) A\left(e_{\alpha}+e_{\beta}\right)$ is primitive [6, Proposition 1, p. 48] and is clearly closed, $\left(e_{\alpha}+e_{\beta}\right) A\left(e_{\alpha}+e_{\beta}\right)$ is a locally compact, primitive $Q$-ring with property $P$, so $\left(e_{\alpha}+e_{\beta}\right)$ $A\left(e_{\alpha}+e_{\beta}\right)$ is a division ring. But it contains nonzero $e_{\alpha}, e_{\beta}$ satisfying $e_{\alpha} e_{\beta}=0$, a contradiction. Thus $\Gamma$ can contain only one element, so $D / J(D)$ is isomorphic to a finite ring. Hence $J(D)$, being closed in $D$, is open in $D$ and thus in $A$, so $A$ is a $Q$-ring.

Lemma 7. If $A$ is an $H_{i}$-ring, $i=1,2,3,4$ and if $A$ is a locally compact division ring, then $A$ is a field.

Proof. If $A$ is discrete and is an $H_{i}$-ring $(i=1,2,3,4)$ then $A$ is commutative [3, Th. $2 ; 4$, Th. $1 ; 3$, Th. $1 ; 1$, Lemma 1].

If $A$ is not discrete, then $A$ has a nontrivial absolute value giving its topology, and $A$ is a finite-dimensional algebra over its center, on which the absolute value is nontrivial [10, Th. 8].

If $A$ is an $H_{1}$-ring and $x$ is nonzero in $A$, then there exists some nonzero $z$ in the center of $A$ such that $|z|<1 /|x|$. Thus $|x z|<1$, so $\lim _{n}(x z)^{n}=0$. Hence for any $y \in A, \lim _{n}(x z)^{n} y-y(x z)^{n}=0$, so as $(x z) y-y(x z)$ is in the closure of $\left\{(x z)^{n} y-y(x z)^{n}: n \geqq 2\right\}, 0=(x z) y-$ $y(x z)=z(x y-y x)$. Hence $x y=y x$, as $z \neq 0$. Thus $A$ is commutative.

If $A$ is an $H_{2}$-ring and if $x, y \in A$ satisfy $x y-y x \neq 0$, then there exists some nonzero $z$ in the center such that $|z|<1 /|x y-y x|$. Thus 
$|(x z) y-y(x z)|<1$, so $\lim _{n}[(x z) y-y(x z)]^{n}=0$. Hence $0=(x z) y-$ $y(x z)=(x y-y x) z$, so $x y-y x=0$ as $z \neq 0$, a contradiction. Thus $A$ is commutative.

Assume that $A$ is an $H_{3}$-ring. As $A$ is a division ring, $A$ is either totally disconnected or connected [7, Th. 2].

Case 1. $A$ is totally disconnected. Then the topology of $A$ is given by a nonarchimedean absolute value. Suppose $A$ is not commutative. Then as $A$ is a finite-dimensional and hence an algebraic extension of its center $C$, there exists some $x \notin C$ having minimal degree $m>1$ over $C$. Let $y$ be arbitrary in $A$, and assume that for no $1 \leqq i \leqq m-1$, does $x^{i} y=y x^{i}$. Hence $x^{i} y-y x^{i} \neq 0,1 \leqq i \leqq m-1$, and we claim $\left\{x^{i} y-y x^{i}: 1 \leqq i \leqq m-1\right\}$ is a linearly independent set over $C$. Suppose $\sum_{i=i}^{m-1} \beta_{i}\left(x^{i} y-y x^{i}\right)=0$, where $\beta_{i} \in C$, and let $z=\sum_{i-i}^{m-1} \beta_{i} x^{i}$. Then $z y=y z$. By the definition of $m$, either $z \in C$ on $z$ has degree $\geqq m$ over $C$. Suppose $z \notin C$. Then $C[x]$ has dimension $m$ over $C$, so $m$ is the degree of $z$ as $z \in C[x]$. Therefore $C[x]=C[z]$, so as $z y=y z$, every element of $C[x]$ commutes with $y$, contrary to our assumption. Thus $z \in C$; let $-\beta_{0}=z$. Then $\sum_{i=0}^{m-1} \beta_{i} x^{i}=0$, so $\beta_{i}=0,0 \leqq i \leqq m-1$ since $\left\{1, x, \cdots, x^{m-1}\right\}$ is linearly independent over $C$.

Since $x$ is algebraic of degree $m$ over the center $C$ of $A$, there exist $\alpha_{i} \in C, 0 \leqq i \leqq m-1$, such that $x^{m}=\sum_{i=0}^{m-1} \alpha_{i} x^{i}$; thus for all $n \geqq m$, there exist $\alpha_{i, n} \in C, 0 \leqq i \leqq m-1$, such that $x^{n}=\sum_{i=0}^{m-1} \alpha_{i, n} x^{i}$. We may also assume that $|x|>1$, since all our assumption on $x$ are true for any $\lambda x, \lambda \in C^{*}$. We note that there is therefore some $r$ such that $|x|^{r} \geqq\left|\alpha_{i}\right|, 0 \leqq i \leqq m-1$.

Since $x^{n}=\sum_{i=0}^{m-1} \alpha_{i, n} x^{i}$,

$$
x^{n} y-y x^{n}=\sum_{i=i}^{m-1} \alpha_{i, n}\left(x^{i} y-y x^{i}\right) ;
$$

so $\lim _{n} x^{n} y-y x^{n}=0$ if and only if $\lim _{n} \alpha_{i, n}=0,1 \leqq i \leqq m-1$.

Since $\left|x^{n}\right| \leqq \max \left\{\left|\alpha_{i, n}\right||x|^{i}: 0 \leqq i \leqq m-1\right\}$, if $\left|\alpha_{i, n}\right|<1,1 \leqq i \leqq$ $m-1$, then $|x|^{n} \leqq\left|\alpha_{0, n}\right|$. Let $r_{0}$ be such that $|x|^{r_{0}}>|x|+1$. Since $\lim _{n} \alpha_{i, n}=0,1 \leqq i \leqq m-1$, there exists $n_{0}>r+r_{0}$ such that $\left|\alpha_{i, n}\right|<1$, for all $n \geqq n_{0}$ and all $i$ such that $1 \leqq i \leqq m-1$. But for any $n>n_{0}$,

$$
\begin{aligned}
x^{n+1} & =\sum_{i=0}^{m-2} \alpha_{i}, x^{i+1}+\alpha_{m-1, n}\left(\sum_{i=0}^{m-1} \alpha_{i} x^{i}\right) \\
& =\alpha_{m-1, n} \alpha_{0}+\sum_{i=1}^{m-1}\left[\alpha_{i-1, n}+\left(\alpha_{m-1, n}\right) \alpha_{i}\right] x^{i},
\end{aligned}
$$

so

$$
\begin{aligned}
\left|\alpha_{1, n+1}\right| & =\left|\alpha_{0, n}+\alpha_{m-1, n} \alpha_{1}\right| \geqq\left|\alpha_{0, n}\right|-\left|\alpha_{m-1, n}\right|\left|\alpha_{1}\right| \\
& \geqq|x|^{n}-\left|\alpha_{1}\right| \geqq|x|^{r+r_{0}}-|x|^{r}=\left|x^{r}\right|\left(|x|^{r_{0}}-1\right)>1 .
\end{aligned}
$$

a contradiction. Hence $A$ is commutative. 
Case 2. $A$ is connected. Then the center $C$ of $A$ contains the real number field $R, A$ is finite-dimensional over $R$, so the degree of each element of $A$ over $R$ is less than or equal to 2, and the topology is given by an absolute value. Suppose $x \notin C$. Then $\operatorname{deg} x=2$; let $x^{2}=\alpha_{1}+\alpha_{2} x$, and for each $n \geqq 2$, let $x^{n}=\alpha_{1, n}+\alpha_{2, n} x$, where $\alpha_{1, n}$, $\alpha_{2, n} \in R$. As before we may assume that $|x|>1$. Let $r$ be such that $|x|^{r}>\max \left\{\left|\alpha_{1}\right|,\left|\alpha_{2}\right|\right\}$. Let $y \in A$ be such that $x y \neq y x$. Then $0=$ $\lim _{n}\left(x^{n} y-y x^{n}\right)=\lim _{n} \alpha_{2, n}(x y-y x)$, so $\lim _{n} \alpha_{2, n}=0$. Let $n_{0}>r$ be such that $\left|\alpha_{2, n}\right|<1$ for all $n \geqq n_{0}$. But if $n \geqq n_{0}$ is such that $|x|^{n}>3$ $|x|^{r}$, then

$$
|x|^{n}=\left|\alpha_{1, n}+\alpha_{2, n} x\right| \leqq\left|\alpha_{1, n}\right|+\left|\alpha_{2, n}\right||x|<\left|\alpha_{1, n}\right|+|x|,
$$

so $\left|x^{n}\right|-|x|<\left|\alpha_{1}, n\right|$. As

$$
\begin{gathered}
x^{n+1}=\alpha_{1, n} x+\alpha_{2, n}\left(\alpha_{1}+\alpha_{2} x\right)=\alpha_{2, n} \alpha_{1}+\left(\alpha_{1, n}+\alpha_{2, n} \alpha_{2}\right) x, \\
\left|\alpha_{2, n+1}\right|=\left|\alpha_{1, n}+\left(\alpha_{1, n}\right) \alpha_{2}\right| \geqq\left|\alpha_{1, n}\right|-\left|\alpha_{2, n}\right|\left|\alpha_{2}\right| .
\end{gathered}
$$

Hence $\left|\alpha_{2, n+1}\right| \geqq\left(|x|^{n}-|x|\right)-|x|^{r} \geqq 3|x|^{r}-|x|^{r}-|x|^{r}=|x|^{r}>1$, a contradiction. Hence $A$ is commutative.

Finally let $A$ be an $H_{4}$-ring. If for all $x$ and $y, \lim _{n} x^{n} y-y x^{n}=0$, then $A$ is an $H_{3}$-ring and so a field; so assume there are $x$ and $y$ in $A$ such that $\lim _{n} x^{n} y-y x^{n} \neq 0$. Let $W=\left\{w \in A: \lim _{n} x^{n} w-w x^{n}=0\right\}$. Clearly $W$ is a division subring of $A$, and since $y \notin W, W$ is a proper division subring. By hypothesis, for all $a \in A$ there is an $r \geqq 1$ such that $a^{r} \in W$; thus $A$ is a field [2, Th. B].

THEOREM 4. All $H_{i}$-rings that are locally compact and semisimple are commutative, $i=1,2,3,4$.

Proof. $P$ is a primitive ideal of such a ring $A$ if and only if $P=(B: A)$ (by definition $(B: A)=\{x \in A: A x \subseteq B\})$ where $B$ is a regular maximal to left ideal [5, Corollary to Proposition 2, p. 7]. Let $e \in A$ be such that $x-e x \in B$ for all $x \in A$. If $x \in(B: A)$, then $e x \in B$, so $x \in B$. Hence $(B: A) \subseteq B$.

If $B$ is closed, then $(B: A)$ is closed for if $\left(x_{\alpha}\right)$ is a directed set of elements of $(B: A)$ converging to $x$, then for all $a \in A, a x_{\alpha} \in B$, whence $a x=\lim a x_{\alpha} \in B$.

As $A$ is semisimple, $(0)=\bigcap\{B: B$ is a closed regular maximal left ideal $\} \supseteqq \bigcap\{P: P$ is a closed primitive ideal $\}[8$, Th. 1]. By Lemma 6 and $7, A / P$ is a field if $P$ is a closed primitive ideal. Thus for all $x, y \in A, x y-y x \in P$, so $x y-y x \in \bigcap\{P: P$ is a closed primitive ideal $\}=(0)$. 


\section{REFERENCES}

1. L. P. Belluce, I. N. Herstein, and S. K. Jain, Generalized commutative rings, Nagoya Math. J. 27 (1966), 1-5.

2. C. Faith, Algebraic division ring extensions, Proc. Amer. Math. Soc. 11 (1960), 43-53.

3. I. N. Herstein, A condition for commutativity of rings, Canad. J. Math. 9 (1957), 583-586.

4. , Two remarks on the commutativity of rings, Canad. J. Math. 7 (1955), 411-412.

5. N. Jacobson, Structure of rings, Math. Soc. Coll. Pub., vol. 37, Providence, Rhode Island, 1956.

6. N. Jacobson and O. Tausky, Locally compact rings, Proc. Nat. Acad. Sci. 21 (1935). 106-108.

7. I. Kaplansky, Locally compact rings, Amer. J. Math. 70 (1948), 447-459.

8. — Locally compact rings, II, Amer. J. Math. 73 (1951), 20-24.

9. _ Locally compact rings, III, Amer. J. Math. 74 (1952), 929-935.

10. - Topological methods in valuation theory, Duke Math. J. 14 (1947), 527-541.

11. Topological rings, Amer. J. Math. 69 (1947), 153-183.

12. Seth Warner, Compact rings, Math. Ann. 145 (1962), 52-63.

Received August 16, 1968. The results in this paper are taken from the author's doctoral dissertation, written at Duke University under Professor Seth Warner.

DUKE UNIVERSITY 


\section{PACIFIC JOURNAL OF MATHEMATICS}

\section{EDITORS}

\author{
H. SAMELSON \\ Stanford University \\ Stanford, California 94305 \\ Richard Pierce \\ University of Washington \\ Seattle, Washington 98105
}

J. DUGUNDJI

Department of Mathematics

University of Southern California

Los Angeles, California 90007

BASIL GORDON*

University of California

Los Angeles, California 90024

\section{ASSOCIATE EDITORS}
E. F. BECKENBACH
B. H. NeUmanN
F. WOLF
K. YoSHIDA

\section{SUPPORTING INSTITUTIONS}

UNIVERSITY OF BRITISH COLUMBIA CALIFORNIA INSTITUTE OF TECHNOLOGY

UNIVERSITY OF CALIFORNIA MONTANA STATE UNIVERSITY

UNIVERSITY OF NEVADA

NEW MEXICO STATE UNIVERSITY

OREGON STATE UNIVERSITY

UNIVERSITY OF OREGON

OSAKA UNIVERSITY

UNIVERSITY OF SOUTHERN CALIFORNIA
STANFORD UNIVERSITY

UNIVERSITY OF TOKYO

UNIVERSITY OF UTAH

WASHINGTON STATE UNIVERSITY

UNIVERSITY OF WASHINGTON

AMERICAN MATHEMATICAL SOCIETY CHEVRON RESEARCH CORPORATION TRW SYSTEMS

NAVAL WEAPONS CENTER 


\section{Pacific Journal of Mathematics}

\section{Vol. 32, No. $1 \quad$ January, 1970}

Robert Alexander Adams, Compact Sobolev imbeddings for unbounded domains ........................................ 1

Bernhard Amberg, Groups with maximum conditions .................. 9

Tom M. (Mike) Apostol, Möbius functions of order k............... 21

Stefan Bergman, On an initial value problem in the theory of two-dimensional transonic flow patterns ................... 29

Geoffrey David Downs Creede, Concerning semi-stratifiable spaces ...... 47

Edmond Dale Dixon, Matric polynomials which are higher

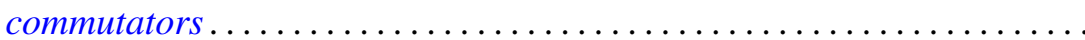

R. L. Duncan, Some continuity properties of the Schnirelmann density.

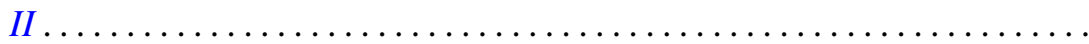

Peter Larkin Duren and Allen Lowell Shields, Coefficient multipliers of $H^{p}$

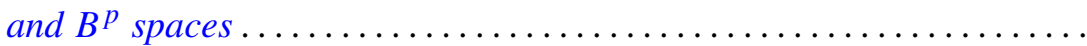

Hector O. Fattorini, On a class of differential equations for vector-valued

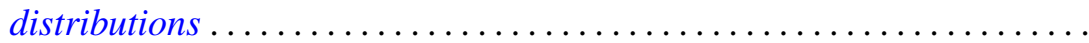

Charles Hallahan, Stability theorems for Lie algebras of derivations. . . . . . 105

Heinz Helfenstein, Local isometries of flat tori ................ 113

Gerald J. Janusz, Some remarks on Clifford's theorem and the Schur

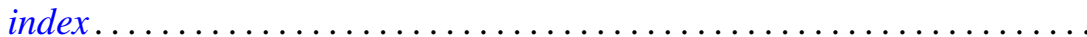

Joe W. Jenkins, Symmetry and nonsymmetry in the group algebras of discrete groups. ...

Herbert Frederick Kreimer, Jr., Outer Galois theory for separable

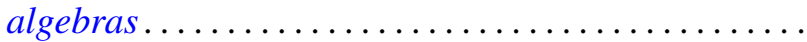

D. G. Larman and P. Mani, On visual hulls

R. Robert Laxton, On groups of linear recurrences. II. Elements of finite order.

Dong Hoon Lee, The adjoint group of Lie groups ...

James B. Lucke, Commutativity in locally compact rings

Charles Harris Scanlon, Rings of functions with certain Lipschitz

$$
\text { properties ............................... }
$$

Binyamin Schwarz, Totally positive differential systems .

James McLean Sloss, The bending of space curves into piecewise helical curves.

James D. Stafney, Analytic interpolation of certain multiplier spaces ...

Patrick Noble Stewart, Semi-simple radical classes.......

Hiroyuki Tachikawa, On left $\mathrm{QF}-3$ rings ...................... 255

Glenn Francis Webb, Product integral representation of time dependent nonlinear evolution equations in Banach spaces.... . . . 